\title{
A gradient approach to localization of deformation. I. Hyperelastic materials
}

\author{
N. TRIANTAFYLLIDIS * and ELIAS C. AIFANTIS \\ MM Program, Dept. of Mechanical Engineering and Engineering Mechanics, \\ Michigan Technological University, Houghton, MI 4993I, USA
}

(Received 1 November 1984; in revised form 13 June 1985)

\begin{abstract}
.
By utilizing methods recently developed in the theory of fluid interfaces, we provide a new framework for considering the localization of deformation and illustrate it for the case of hyperelastic materials. The approach overcomes one of the major shortcomings in constitutive equations for solids admitting localization of deformation at finite strains, i.e. their inability to provide physically acceptable solutions to boundary value problems in the post-localization range due to loss of ellipticity of the governing equations. Specifically, strain-induced localized deformation patterns are accounted for by adding a second deformation gradient-dependent term to the expression for the strain energy density. The modified strain energy function leads to equilibrium equations which remain always elliptic. Explicit solutions of these equations can be found for certain classes of deformations. They suggest not only the direction but also the width of the deformation bands providing for the first time a predictive unifying method for the study of pre-and post-localization behavior. The results derived here are a three-dimensional extension of certain one-dimensional findings reported earlier by the second author for the problem of simple shear.
\end{abstract}

\section{Introduction}

In two recent papers Aifantis and Serrin [1-2] examined the equilibrium form of fluid interfaces and microstructures by introducing second density gradients in the dependence of interfacial stress, deriving a nonlinear partial differential equation for the interfacial density, and finding all possible solutions of this equation in one dimension. They were transitions (monotone solutions with distinct limits at infinity), reversals (symmetric solutions about a single maximum or minimum with a common definite limit at infinity), and oscillations (periodic solutions with finite but not definite limits at infinity). Physically, they were identified with liquid-vapor interfaces, thin films, and layered structures, respectively.

The relevance of these solutions to the localization of solid microstructures and their relation to the localization of macroscopic deformation was discussed by Aifantis [3-7]. It was shown that the density of dislocations, microvoids, and other material microstructures can generally be specified by equations analogous to those determining the density of fluid microstructures. Moreover, elimination of the microstructural variables (internal variables) from the constitutive equations can generate higher order gradients in the stress-strain relations [6].

* Permanent address: Dept. of Aerospace Engineering, The University of Michigan, Ann Arbor, MI 48109 
For the one-dimensional problem of simple shear [6,7], in particular, the expression between shear stress and shear strain is similar to the relation between interfacial stress and fluid density in [1-2]. The form of the corresponding equilibrium equation is also analogous to that studied in [1,2] but the only solution of relevance here is the reversal since the strain has the same values at both ends. It thus turns out [7] that unlike the usual approach, the strain distribution is continuous with its maximum at the center of the shear band and its shape determining the size of this localized zone. The direction of the band was found to be parallel to the applied shear as in the classical approach but the onset of localization stress was found to be lower than the one predicted by classical analysis, in qualitative agreement with observations.

The motivation for the present paper was to find generalizations of the above one-dimensional results for simple shear and examine the implications of the gradient approach to localization within a three-dimensional context. We were interested to compare the predictions of the gradient and the classical approaches and find a position for the loss of ellipticity condition of the classical approach within the structure of the gradient approach. Moreover, we wished to provide an estimate of the size of the narrow localized zone, a task which remains impossible within the classical approach, if a preexisting imperfection is not assumed. To accomplish clarity and maintain simplicity, we illustrate the method within a framework for hyperelastic materials. For completeness, however, we give below a brief review of the main results of the classical approaches as they relate to the present gradient approach.

The connection between localization of deformation and the loss of ellipticity in the governing equilibrium equations has been proposed in the context of elasticity by Hadamard [8] and in the case of non-elastic materials by Thomas [9], Hill [10] and Mandel [11]. The characteristic surfaces in this approach indicate the location and shape of the localized deformation zones. Considerable effort has been recently devoted to the study of localization phenomena and their dependence on the assumed constitutive models. Brief reviews on the subject can be found in the works of Knowles and Sternberg [12] for hyperelastic materials and Rice [13] for inelastic ones.

The loss of ellipticity in the equilibrium equations approach, although adequate for predicting the zone direction and critical stress level, leaves the localized zone size unspecified. An effort to remedy the situation by introducing favorably oriented imperfections in the form of weak zones in the material has been developed in the context of localized necking in thin sheets (e.g. Marciniak and Kuczynski [14]). This method, however, is descriptive rather than predictive, it has the serious drawback of imposing artificial inhomogeneities, and the disadvantage of requiring unrealistic and physically non-observable imperfection orientations and amplitudes.

The aforementioned absence of any characteristic length for the localized strain zone at the loss of ellipticity of the equilibrium equations, is the reason for the critical dependence of finite element calculations in related boundary value problems on the employed finite element mesh size. In addition, the post-localization calculations for such solids presents a high degree of difficulty due to mesh size related numerical problems and the results, whenever possible, are often physically unacceptable. More details on these aspects can be found in the article by Tvergaard, Neeldleman and Lo [15] and references quoted therein.

The fact that there is plenty of experimental evidence for the development of small but finite size localized deformation zones in sufficiently strained initially uniform 
materials * points to the need for an approach to localization which will automatically generate in a predictive and natural way localized deformation solutions at large strains. Such a procedure is possible by introducing higher-order deformation gradients in the constitutive equations as illustrated by Aifantis and Serrin $[1,2]$ for the case of fluid microstructures and Aifantis [6,7] for the case of simple shear.

In order to simplify our task, attention in this work will be focussed only on hyperelastic materials, whose equilibrium equations lose ellipticity at finite strains. It will be shown that a simple modification in the strain energy density function describing these materials produces the desirable localized solutions. This modification is accomplished by the addition of higher order deformation gradients ** in the strain energy density, much in the spirit of some recent work in the microscopic theory of phase transformations (e.g. Aifantis [16] and Alexiades and Aifantis [17]). In this connection, we also point out that although higher order gradients have been familiar objects to the workers in continuum mechanics for quite some time (e.g. Toupin $[18,19]$, Mindlin [20,21], Green and Rivlin [22]), their use has been rather formal and their implications have not been investigated in the non-linear regime, as in the present case.

The plan of the paper is as follows: In Section 2 we give the form of the modified strain energy function for hyperelastic materials and the resultant equilibrium equations which now remain elliptic throughout the process of deformation. Section 3 contains the analysis of the emergence of localized deformation bands in an infinite medium. It is shown that the direction of the band is determined by the loss of ellipticity condition for the unmodified material as in the classical analyses. In contrast to the classical results, however, a prediction for the width of the band is now possible and an explicit formula is provided. Finally, in Section 4 we apply our findings to the localization behavior of a particular class of hyperelastic materials (Blatz-Ko materials) for which explicit analytic solutions are possible.

\section{Modified hyperelastic materials}

We consider a continuum body occupying a region $V$ in space with boundary $\partial V$. A full Lagrangian description of the body's equilibrium equations is adopted here with the stress-free configuration chosen to be the reference one. Material points in the solid are identified by their position vector $\boldsymbol{X}$ in the reference state while the corresponding current position vector is denoted by $\boldsymbol{x}(\boldsymbol{X})$. The second rank tensor $\boldsymbol{F}=\nabla \boldsymbol{x}$ is called the deformation gradient tensor and satisfies $\operatorname{det} F>0$ on $V$. For the sake of simplicity Cartesian coordinates will be used in this presentation and we will interchange direct and indicial notation according to convenience.

As mentioned earlier, attention is confined to hyperelastic materials whose equilibrium equations lose ellipticity at adequately large levels of strain i.e. materials whose

* I.e. homogeneous materials with uniform properties and no preassumed inclusions or imperfections of the size and shape of the subsequently developed localized deformation bands.

** These higher order gradients may be thought of as a macroscopic manifestation of the various microprocesses including thermal ones that may occur at the microsale. 
strain energy density per unit reference volume $w(\boldsymbol{F})$ satisfies the condition *

$$
\operatorname{det}\left[L_{i j k l} n_{j} n_{l}\right] \leqslant 0, \quad L_{i j k l} \equiv \partial^{2} w / \partial F_{i j} \partial F_{k l} \text {, }
$$

for some unit vector $\boldsymbol{n}$ and some deformation gradient $\boldsymbol{F} \neq 1$. For hyperelastic materials satisfying (2.1) Knowles and Sternberg [23] have shown the possibility of solutions with discontinuous deformation gradients across surfaces with normal $\boldsymbol{n}$. In this connection, we remark that for rate independent elastoplastic materials also, condition (2.1), with $\boldsymbol{L}$ interpreted as the incremental moduli tensor relating the rate of the nominal stress tensor to the rate of the deformation gradient tensor, is necessary for the existence of solutions with localized shear deformation zones perpendicular to $n$ (e.g. Rice [13], Hill and Hutchinson [24]).

Motivated by the desire to introduce, in accordance with experimental observations, a physical length scale for the zone of localized deformations in materials satisfying (2.1), we propose to modify the strain energy function of such materials by incorporating second gradients of deformation. This will eventually enable us to study post-localization behavior in a manner free of the inconsistencies discussed in the introduction. Although the existence of potentials for unstable regions and the generality of the variational approach as compared to a purely mechanical approach has been questioned by Aifantis and Serrin [1,2], a common domain of validity has been found by Aifantis [16] and a related discussion has been given by Alexiades and Aifantis [17]. In this last paper Alexiades and Aifantis [17] also address the existence of minimizers and the stability of the solutions derived by Aifantis and Serrin [1,2], questions which are also essential for the present study.

These aspects are not pursued here in depth, since we are mainly interested in providing only a simple illustration of the proposed mechanism for the determination of deformation band sizes. In the same spirit, we also adopt the framework of finite strain elasticity theory due to its clarity and relative simplicity as compared to some of the more complicated constitutive models for inelastic solids. Finally, and for the same reasons, we consider infinite domains so that we can dispense with questions concerned with the nature and interpretation of the correct boundary conditions, as well as the appropriate form of the stress tensor and the associated tractions. Different methods in arriving at appropriate forms of stress tensors (both asymmetric and symmetric) for gradient-sensitive materials have been suggested by various authors including Toupin [18-19], Aifantis [16], Dunn and Serrin [25] and Silhavy and Aifantis [26]. Their results will be useful in future discussions of finite bodies and boundary value problems related to the gradient-approach to localization.

A modified hyperelastic material is defined by a strain energy density function $\hat{w}$ of the form

$$
\hat{w}=w(\boldsymbol{F})+\kappa h(\boldsymbol{F}, \boldsymbol{G}) ; \quad \boldsymbol{G} \equiv \nabla \boldsymbol{F} ; \quad \kappa>0,
$$

where $h$ is homogeneous and quadratic ** with respect to the third rank tensor $G\left(G_{i j k}=\partial x_{i} / \partial X_{j} \partial X_{k}\right), \kappa$ is a positive constant (singled out for convenience) whose

* If $a$ is a unit vector, condition (2.1) implies the loss of strong ellipticity, i.e. $L_{i j k l} a_{i} a_{k} n_{j} n_{l} \leqslant 0$ or $\left[\partial^{2} w / \partial F_{i j} \partial F_{k l}\right] a_{i} a_{k} n_{j} n_{l} \leqslant 0$.

** I.e., $h(\boldsymbol{F}, \lambda \boldsymbol{G})=\lambda^{2} h(\boldsymbol{F}, \boldsymbol{G})$ for all reals $\lambda \neq 0$ and, in particular, $\partial^{3} h / \partial G_{i j k} \partial G_{l m n} \partial G_{p q r}=0$. In actuality, it turns out that this assumption can be relaxed and we only need for $h$ to be homogeneous of degree two and convex in $\boldsymbol{G}$ in order for our results to be still valid, i.e. $\left(\partial^{2} h / \partial G_{i j k} \partial G_{m n r}\right) M_{i j k} M_{m n r} \geqslant 0$ for all third rank tensors $\boldsymbol{M}$, symmetric in their last two indices. 
physical meaning will be discussed later, and $w(\boldsymbol{F})$ is the energy density of the unmodified material which satisfies (2.1). Some additional requirements will be imposed on $h$ so as to ensure the non-negativeness and frame indifference of $\hat{w}$. Thus, $h \geqslant 0$ with the equality holding for $\boldsymbol{F}=\boldsymbol{Q}$ and $\boldsymbol{Q}\left(Q_{i j}\right)$ any orthogonal tensor constant in $\boldsymbol{X}$; in addition $h\left(\partial x_{i} / \partial X_{j}, \partial^{2} x_{k} / \partial X_{l} \partial X_{m}\right)=h\left(\partial x_{i}^{*} / \partial X_{j}, \partial^{2} x_{k}^{*} / \partial X_{l} \partial X_{m}\right)$ for $x_{i}^{*}=$ $Q_{i j} x_{j}$. Moreover, in the case of an isotropic material it will be additionally required that $h\left(\partial x_{i} / \partial X_{j}, \partial^{2} x_{k} / \partial X_{l} \partial X_{m}\right)=h\left(\partial x_{i} / \partial X_{j}^{*}, \partial^{2} x_{k} / \partial X_{l}^{*} \partial X_{m}^{*}\right)$ for $X_{i}^{*}=Q_{j i} X_{j}$. The frame indifference and material symmetry requirements imposed on $h$ (assuming of course that similar ones already hold for $w$ ) are a straightforward application of standard definitions in continuum mechanics (e.g. Truesdell and Toupin [27], as well as Cross [28] and Bowen [29] for higher order materials).

Before proceeding to the study of localized deformations in hyperelastic materials whose strain energy densities are given by (2.2), it is instructive to present the form of the corresponding equilibrium equations and of the associated boundary conditions. If $b$ denotes the body force per unit reference volume, the potential energy functional $\Pi(\boldsymbol{x})$ is defined as usual by

$$
\Pi(\boldsymbol{x})=\int_{V} \hat{w} \mathrm{~d} V-\int_{V} \boldsymbol{b} \cdot(\boldsymbol{x}-\boldsymbol{X}) \mathrm{d} V-\text { Boundary Terms. }
$$

On computing the first variation $\delta \Pi$ of (2.3) and setting it equal to zero we obtain the pointwise equilibrium equations in $V(\delta \Pi=0)$ in the form

$$
\frac{\partial}{\partial X_{j}}\left[-\kappa \frac{\partial}{\partial X_{k}}\left(\frac{\partial h}{\partial G_{i j k}}\right)+\kappa \frac{\partial h}{\partial F_{i j}}+\frac{\partial w}{\partial F_{i j}}\right]+b_{i}=0,
$$

while at the boundary, the following quantities are assumed to be prescribed

$$
\begin{aligned}
& \left(\kappa \frac{\partial h}{\partial G_{i j k}} n_{k} \text { or } \frac{\partial x_{i}}{\partial X_{j}}\right) \quad \text { and } \\
& \left(\left[-\kappa \frac{\partial}{\partial X_{k}}\left(\frac{\partial h}{\partial G_{i j k}}\right)+\kappa \frac{\partial h}{\partial F_{i j}}+\frac{\partial w}{\partial F_{i j}}\right] n_{j} \text { or } x_{i}\right),
\end{aligned}
$$

where $n$ is the reference outward unit normal to $\partial V$. For reasons explained in the introduction, the physical interpretation of the boundary conditions and their relation to the appropriate tractions will be addressed in the future.

A closer examination of (2.4) reveals it to be a quasilinear differential equation in $\boldsymbol{x}$ with the highest order term being

$$
\kappa \frac{\partial^{2} h}{\partial G_{i j k} \partial G_{l m n}} \frac{\partial^{4} x_{l}}{\partial X_{m} \partial X_{n} \partial X_{k} \partial X_{j}}+\text { Lower Order Terms }=0
$$

In view of the assumed properties of $h$ we have

$$
h(\boldsymbol{F}, \boldsymbol{G})=\frac{1}{2} G_{i j k} G_{l m n} B_{i j k l m n}(\boldsymbol{F}) ; \quad B_{i j k l m} \equiv \frac{\partial^{2} n}{\partial G_{i j k} \partial G_{l m n}},
$$

for all possible * third rank tensors $G$. Thus, from the assumed positiveness of $h$ for

\footnotetext{
* Obviously, the higher order tensors $G, B$, etc., have certain inherent symmetries which, however, are not essential in the present development.
} 
$G \neq 0$ and (2.7), the fourth order form associated with the partial differential operator on $\boldsymbol{x}$ in (2.6) is positive definite

$$
\kappa B_{i j k l m n}(\boldsymbol{F}) a_{i} a_{l} n_{j} n_{k} n_{m} n_{n}>0
$$

for all unit vectors $a$ and $n$. Hence, the equilibrium equations (2.4) or equivalently (2.6), are strongly elliptic * at all stages of deformation, thus excluding solutions with discontinuous deformation gradients (at least up to fourth order) since no real characteristic surfaces exist.

\section{Finite localized deformation zones}

In order to follow the development of a localized zone of deformation, attention is focussed on a special class of deformations, namely those obeying

$$
x_{i}=\stackrel{\circ}{F}_{i j} X_{j}+a_{i} f(z), \quad z \equiv n_{k} X_{k},
$$

where $\stackrel{\circ}{F}$ is a constant (with respect to $X$ ) second rank tensor with positive eigenvalues, $\boldsymbol{a}, \boldsymbol{n}$ are constant unit vectors and $f(z)$ is an adequately smooth function of $z$. The above class of deformations corresponds to a localized deformation zone of shape $f(z)$ in a direction perpendicular to $\boldsymbol{n}$ and of amplitude $\boldsymbol{a}$ superimposed on a uniform strain field with deformation gradient $\stackrel{\circ}{\boldsymbol{F}}$.

On using (3.1) and primes to denote derivatives of a function with respect to its argument, one obtains from (2.2) and (2.3) that the associated potential energy in the absence of body forces is given in this case by

$$
\Pi(f)=\int_{V}\left[\frac{\kappa}{2}\left(g^{\prime}\right)^{2} \hat{h}(g)+w(g)\right] \mathrm{d} V-B . T . ; \quad g \equiv f^{\prime},
$$

where $g$ is the derivative of the shape function $f, w(g) \equiv w(\stackrel{\circ}{\boldsymbol{F}}+g \boldsymbol{a} \otimes \boldsymbol{n}), \hat{h}(g) \equiv 2 h(\stackrel{\circ}{\boldsymbol{F}}$ $+g \boldsymbol{a} \otimes \boldsymbol{n}, \boldsymbol{a} \otimes \boldsymbol{n} \otimes \boldsymbol{n})=B_{i j k l m n}(\dot{\boldsymbol{F}}+g \boldsymbol{a} \otimes \boldsymbol{n}) a_{i} a_{i} n_{j} n_{k} n_{m} n_{n}$ and B.T. denotes the boundary terms in the potential energy [see (2.3)].

On seeking extrema of the potential energy in (3.2) with respect to $f$ we obtain the following nonlinear ordinary differential equation of equilibrium in terms of $g(z)$

$$
\kappa \hat{h}(g) g^{\prime \prime}+\frac{\kappa}{2} \frac{\mathrm{d} \hat{h}}{\mathrm{~d} g}\left(g^{\prime}\right)^{2}=\frac{\mathrm{d} w}{\mathrm{~d} g}-\bar{c},
$$

where $\bar{c}$ is a constant to be subsequently specified. The same result would have been obtained by introducing (3.1) into the general equilibrium equations (2.4). One can observe, by taking the deformation gradient in (3.1), that the imposed strain on the solid is increasing or decreasing proportionally to $g$ and hence for simplicity in terminology $g$ is to be hereafter referred to as the strain parameter.

Next we assume that the medium is infinite and that the strain at infinity (along the $\boldsymbol{n}$ direction) is constant i.e.

$$
g(z) \rightarrow g_{1} \quad \text { as } \quad z \rightarrow \pm \infty,
$$

Of interest here are localized strain type of solutions for $g$, i.e. bell shaped solutions that present a maximum say $g_{2}$ at a single point in $\mathbb{R}$ and which at infinity approach 
asymptotically $g_{1}\left(g_{1}<g_{2}\right)$. Such types of solutions of (3.3) satisfying (3.4) do exist and were called "reversals" by Aifantis and Serrin [1,2] who studied the behavior of a general class of nonlinear ordinary differential equations of the form $a(g) g^{\prime \prime}+$ $b(g)\left(g^{\prime}\right)^{2}=c(g)-\bar{c}$ in the context of a mechanical theory for fluid microstructures.

A necessary (and, as it will be shown later, sufficient for our case) condition for reversals to exist (at some point of the medium) is

$$
\left.\frac{\mathrm{d} w}{\mathrm{~d} g}\right|_{g_{1}}>\left.\frac{\mathrm{d} w}{\mathrm{~d} g}\right|_{g_{2}} \text { for some } g_{1}<g_{2},
$$

or equivalently, for adequately smooth $w$ there exists a $g\left(g_{1}<g<g_{2}\right)$ such that

$$
\frac{\mathrm{d}^{2} w}{\mathrm{~d} g^{2}} \leqslant 0 \Rightarrow\left[\frac{\partial^{2} w}{\partial F_{i j} \partial F_{k l}}\right] a_{i} a_{k} n_{j} n_{l} \leqslant 0,
$$

which is precisely * the loss of strong ellipticity condition given in (2.1). Hence the loss of strong ellipticity in $w$ for the class of deformations adopted in (3.1) ensures the existence of localized deformation solutions. From the same reasoning it follows that as long as the unmodified material is strongly elliptic, i.e. the matrix defined in (2.1) is positive definite $\left[a_{i} n_{j}\left(\partial^{2} w / \partial F_{i j} \partial F_{k l}\right) a_{k} n_{l}>0\right]$ for all possible deformation gradients, then no localized deformation solutions are possible in the proposed modified material.

Appropriating results from Aifantis and Serrin [1,2], the solution to (3.3) and (3.4) is found to be (see also [17])

$$
z=z_{0}+\kappa^{1 / 2} \int_{g\left(z_{0}\right)}^{g(z)}\left\{\hat{h}(g) / 2\left[w(g)-w\left(g_{1}\right)-\frac{\mathrm{d} w}{\mathrm{~d} g_{1}}\left(g-g_{1}\right)\right]\right\}^{1 / 2} \mathrm{~d} g,
$$

with $z_{0}$ being arbitrary and the minimum and maximum strain parameters $g_{1}$ and $g_{2}$ related by

$$
w\left(g_{2}\right)-w\left(g_{1}\right)=\left(\mathrm{d} w / \mathrm{dg}_{1}\right)\left(g_{2}-g_{1}\right) .
$$

In view of the conditions at infinity (3.4), it also turns out that the constant $\bar{c}$ in (3.3) is exactly $\left(\mathrm{d} w / \mathrm{d} g_{1}\right)$, i.e. the derivative of $w$ with respect to $g$ evaluated at $g=g_{1}$. The form of the solution (3.7) is valid for $-\infty<z<z_{2}$ with $g\left(z_{2}\right)=g_{2}$, is symmetric about $z_{2}$, and is depicted in Figure 1; while the Maxwell condition (3.8) is illustrated in Figure 2. The graph of $\mathrm{d} w / \mathrm{d} g$ versus $g$ can have either of the forms shown in Figure 2 and the line $\bar{c}=\mathrm{d} w / \mathrm{d} g_{1}$ determining the maximum value $g_{2}$ is drawn such that to cut equal areas above and below.

The obtained reversal solution for $g$ corresponds to the following physical situation: Consider a deformation process in which the strain parameter at infinity has the value $g_{1}$. If the maximum strain parameter $g_{2}$ in the medium is such that $g_{2}<g_{m}$ where $g_{m}$ satisfies $\mathrm{d}^{2} w / \mathrm{d} g_{m}^{2}=0$, no reversal solution is possible (we are tacitly assuming that $\mathrm{d}^{2} w / \mathrm{dg}^{2}>0$ for $0 \leqslant g<g_{m}$ ) and $g=g_{1}$ is the solution to (3.3). Once the maximum strain parameter exceeds $g_{m}$, i.e. $g_{2}>g_{m}$, then a localized deformation (reversal) type solution is possible. For increasing $g_{2}$ (and assuming $\mathrm{d}^{2} w / \mathrm{d} g^{2}<0$ for $g_{2} \geqslant g>g_{m}$ ) it follows from (3.8) that the strain at infinity $g_{1}$ decreases. This is in agreement with observations in highly strained solids where unloading outside the localized zone

\footnotetext{
* In actuality, $(3.6)_{2}$ is implied by (2.1).
} 


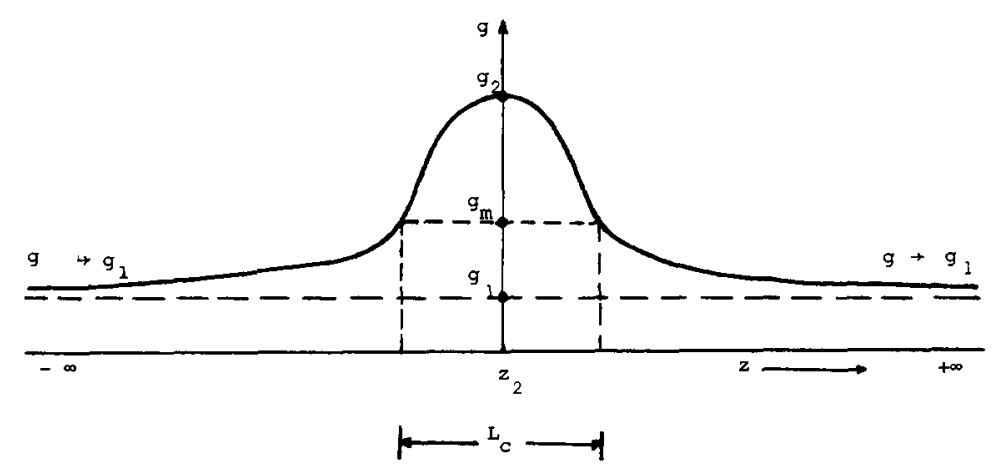

Figure 1
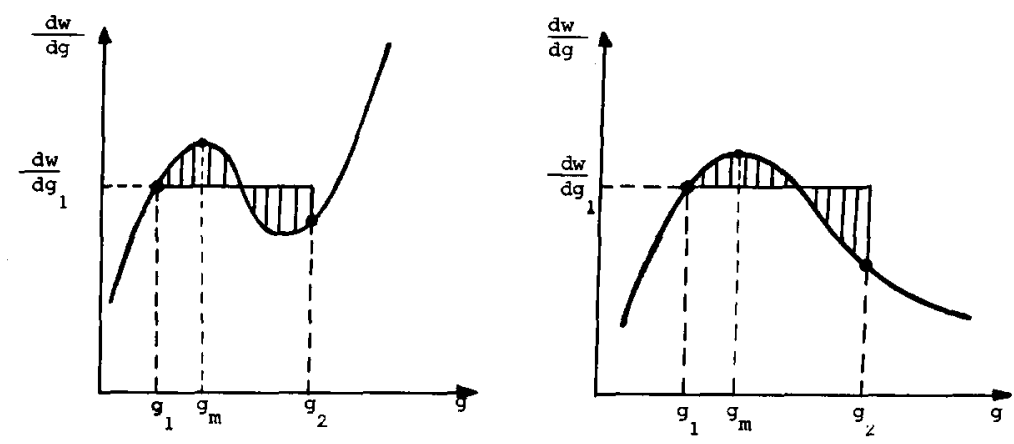

Figure 2

ollows the onset of a localized area of deformation. Some interesting numerical alculations for this phenomenon in the case of hyperelastic and elastic-plastic materials have been presented by Tvergaard, Needleman and Lo [15] where post-localization calculations using the finite element method have also been made.

The characteristic length $L_{c}$ of the localized deformation zone [a quantity which in all previous analytical or numerical studies depended on the size of the assumed imperfections and/or the size of the finite element mesh] is hereby defined to be the distance along $n$ between the two points for which $g=g_{m}$ and hence from (3.7)

$$
L_{c}=2 \kappa^{1 / 2} \int_{g_{m}}^{g_{2}}\left\{\hat{h}(g) / 2\left[w(g)-w\left(g_{1}\right)-\frac{\mathrm{d} w}{\mathrm{~d} g_{1}}\left(g-g_{1}\right)\right]\right\}^{1 / 2} \mathrm{~d} g .
$$

The physical significance of the constant $\kappa$ in the model (2.2) is now apparent since from (3.9) the width of the localized deformation zone is proportional to $\kappa^{1 / 2}$. Obviously for the case of the unmodified material, $\kappa=0, \ldots$, and hence the size of the localization zone shrinks to zero.

\section{Results for modified Blatz-Ko materials}

A hyperelastic constitutive model proposed some time ago by Blatz and Ko [31] became the basis for subsequent investigations (e.g. Knowles and Sternberg [12], [32] 
and references quoted therein) pertaining to the material's loss of ellipticity at finite levels of strain. The strain energy density function $w$ for the Blatz-Ko material is given by

$$
w_{\mathrm{BK}}=\frac{\mu}{2}\left[\frac{\mathrm{II}_{c}}{\mathrm{III}_{c}}+2 \sqrt{\mathrm{III}_{c}}-5\right] \text {, }
$$

where $\mathrm{I}_{c}, \mathrm{II}_{c}, \mathrm{III}_{c}$ are the three invariants of the right Cauchy-Green tensor $\boldsymbol{C}=\boldsymbol{F}^{T} \boldsymbol{F}$ and $\mu$ is the material's shear modulus at infinitesimal deformations. Using the above material as the unmodified one, we will investigate the localization of deformation properties of two modified versions called $M 1$ and $M 2$ of the type proposed in (2.2) with strain energy densities

$$
\left.\begin{array}{ll}
M 1: & \hat{w}_{1}=w_{\mathrm{BK}}+\frac{\kappa}{2}\left(\partial F_{i j} / \partial X_{k}\right)\left(\partial F_{i j} / \partial X_{k}\right) \\
M 2: & \hat{w}_{2}=w_{\mathrm{BK}}+\frac{\kappa}{2}\left(\partial C_{i j} / \partial X_{k}\right)\left(\partial C_{i j} / \partial X_{k}\right) .
\end{array}\right\}
$$

It can easily be verified from the discussion in Section 2.1 that both $\hat{w}_{1}$ and $\hat{w}_{2}$ are frame indifferent as well as isotropic. For analytical simplicity of the resulting formulae attention will be restricted to the class of deformations in (3.1) with $\stackrel{\odot}{F}=1$. It should be noted at this point that based on our analysis in the previous section, the choice of $\stackrel{\boldsymbol{F}}{\boldsymbol{a}} \boldsymbol{a}, \boldsymbol{n}$ is only arbitrary. A more realistic choice in this case would have been $\stackrel{\circ}{F}_{i j}=\lambda_{i} \delta_{i j}$ (no sum) where the principal stretches $\lambda_{i}$ are in the neighborhood of the ellipticity boundary of the Blatz-Ko material in $\lambda_{i}$ space, and $a, n$ are the amplitude and characteristic direction respectively for the loss of ellipticity at the aforementioned

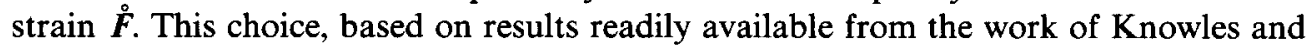
Sternberg [32], would provide information for the growth of a localized deformation zone $^{*}$ at the onset of the loss of ellipticity failure of the material, while the simpler choice adopted here provides information only for a restricted class of deformations. The considerable algebraic simplification as well as the similarity of the resulting analysis has prompted the approach adopted in this discussion.

With (3.1), $\mathrm{II}_{c}$ and $\mathrm{III}_{c}$ are found to be

$$
\begin{aligned}
& \mathrm{II}_{c}=\frac{1}{2}\left[\left(C_{k k}\right)^{2}-C_{i j} C_{i j}\right]=3+4\left(a_{i} n_{i}\right) g+\left[1+\left(a_{i} n_{i}\right)^{2}\right] g^{2}, \\
& \mathrm{III}_{c}=\operatorname{det} C_{i j}=\left[1+\left(a_{i} n_{i}\right) g\right]^{2},
\end{aligned}
$$

and consequently the energy density for the Blatz-Ko material $w_{\mathrm{BK}}$ assumes the form

$$
w_{\mathrm{BK}}(g)=\mu\left[\left(\frac{1}{2 \phi^{2}}+y\right)\left(1-\frac{1}{y}\right)^{2}\right] ; \quad y \equiv 1+g \phi, \quad \phi \equiv a_{i} n_{i} .
$$

Accordingly, the strain parameter $g_{m}$ corresponding to the first loss of ellipticity in the class of deformations (3.1), i.e. $\mathrm{d}^{2} w_{\mathrm{BK}} / \mathrm{d} g_{m}^{2}=0$, is given by

$$
g_{m}=\frac{1+2 \phi^{2}}{2 \phi\left(1-\phi^{2}\right)}, \quad\left[y_{m}=\frac{3}{2\left(1-\phi^{2}\right)}\right] \text {. }
$$

\footnotetext{
* This need not be an actually observed localized zone as experiments do not reveal it for hyperelastic materials, although the corresponding constitutive equation exhibits this possibility.
} 


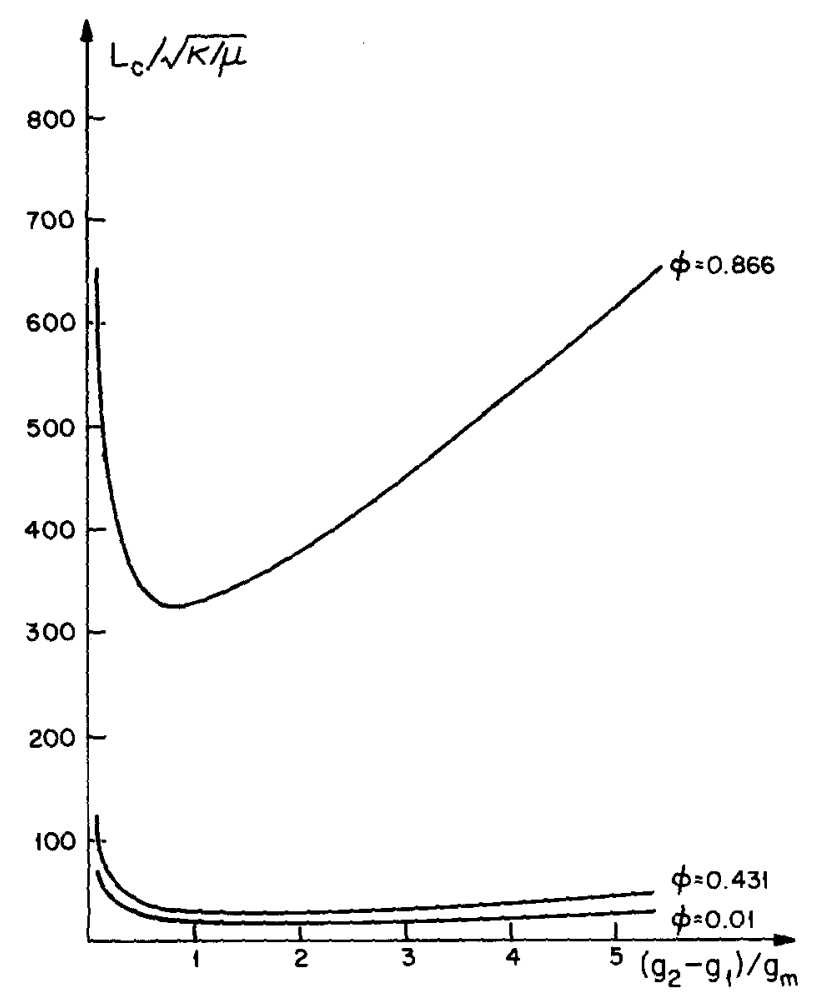

Figure 3

The relation between the maximum strain parameter $g_{2}$ (or $y_{2}$ ) and the strain parameter at infinity $g_{1}$ (or $y_{1}$ ) is found from (3.8) to be

$$
\begin{aligned}
\left(\frac{1}{2 \phi^{2}}+y_{2}\right)\left(1-\frac{1}{y_{2}}\right)^{2}-\left(\frac{1}{2 \phi^{2}}+y_{1}\right)\left(1-\frac{1}{y_{1}}\right)^{2}= & \left(\frac{1}{\left(\phi y_{1}\right)^{2}}+\frac{1}{y_{1}}+1\right)\left(1-\frac{1}{y_{1}}\right) \\
& \times\left(y_{2}-y_{1}\right),
\end{aligned}
$$

and hence the characteristic length $L_{c}$ of the localized deformation zone for materials $M 1$ and $M 2$ is from (3.9) [using also (3.1) and (4.2)]

$$
\left.\begin{array}{l}
\text { M1: } L_{c}=\frac{2}{\phi}\left(\frac{\kappa}{\mu}\right)^{1 / 2} \int_{y_{m}}^{y_{2}}\left(\frac{0.5}{H}\right)^{1 / 2} \mathrm{~d} y, \\
M 2: \quad L_{c}=\frac{2}{\phi}\left(\frac{\kappa}{\mu}\right)^{1 / 2} \int_{y_{m}}^{y_{2}}\left(\frac{1+\phi^{2}+2(y-1)\left\{2+\left[(y-1) / \phi^{2}\right]\right\}}{H}\right)^{1 / 2} \mathrm{~d} y,
\end{array}\right\}
$$

where

$$
\begin{aligned}
H \equiv & \left(\frac{1}{2 \phi^{2}}+y\right)\left(1-\frac{1}{y}\right)^{2}-\left(\frac{1}{2 \phi^{2}}+y_{1}\right)\left(1-\frac{1}{y_{1}}\right)^{2} \\
& -\left(\frac{1}{\phi^{2} y_{1}^{2}}+\frac{1}{y_{1}}+1\right)\left(1-\frac{1}{y_{1}}\right)\left(y-y_{1}\right) .
\end{aligned}
$$




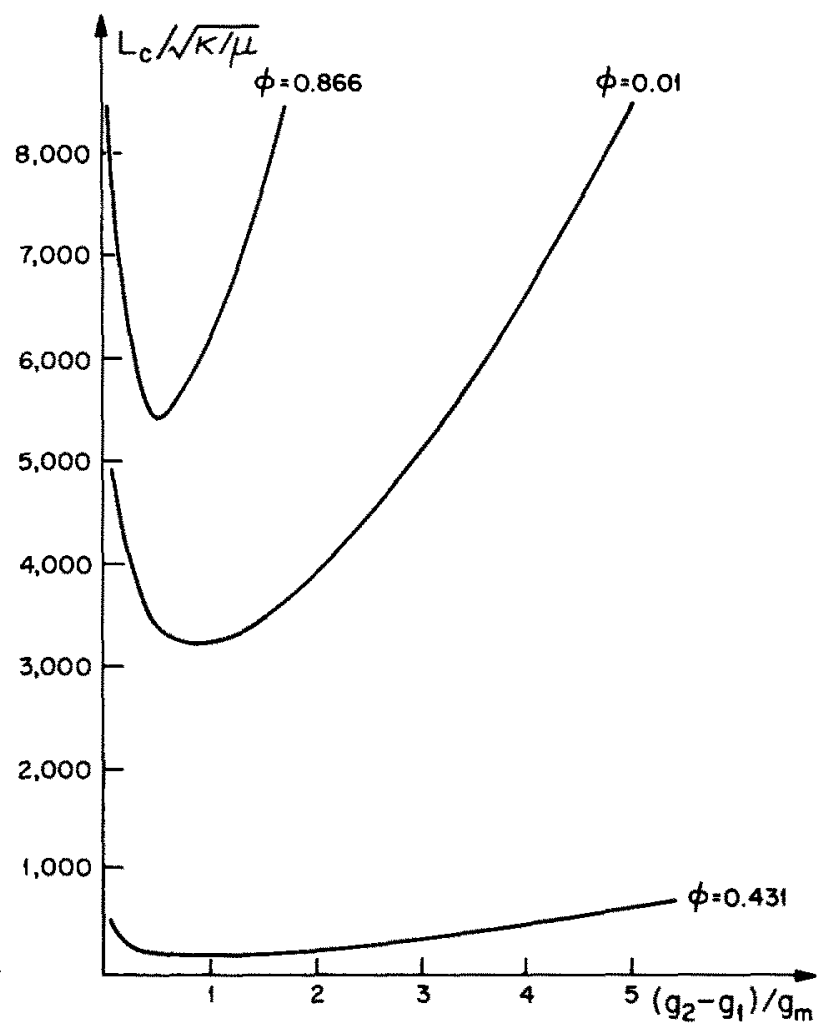

Figure 4

The results from (4.7) are depicted in Figures 3 and 4 for the $M 1$ and $M 2$ materials respectively, where the dimensionless wavelength $L_{c} / \sqrt{\kappa / \mu}$ is plotted versus a strain concentration parameter $\left(g_{2}-g_{1}\right) / g_{m}$ [defined to be the difference between the maximum $\left(g_{2}\right)$ and minimum $\left(g_{1}\right)$ strain parameters over the critical value of the same parameter that corresponds to the onset of localization $\left.\left(g_{m}\right)\right]$. For both materials the calculations are performed for three different values of the parameter $\phi$, the inner product between the amplitude direction $\boldsymbol{a}$ and the characteristic direction $n$, namely $\phi=\cos (30)^{\circ}=0.866, \phi=\cos (64.4)^{\circ}=0.431$ and $\phi=\cos (89.4)^{\circ}=0.01$. The case $\phi=$ 0.431 (more precisely $\phi=\sqrt{\sqrt{33}-5} / 2$ ) corresponds to the minimum value of the strain parameter $g_{m}$ for which localization starts as one can easily see from (4.5) by minimizing with respect to $\phi$.

For both materials, the characteristic length of localization $L_{c}$ drops very rapidly from $\infty$ to its minimum possible value, for values of the strain concentration parameter $\left(g_{2}-g_{1}\right) / g_{m}$ between 0 [corresponding to the onset of the localization instability $\left.\left(g_{1}=g_{2}=g_{m}\right)\right]$ and approximately 1 , and then increases again. The smallest localization length for material $M 1$ is found for $\phi=0$, i.e. for the case where the amplitude vector $\boldsymbol{a}$ is normal to characteristic direction vector $\boldsymbol{n}$ and hence the corresponding deformation is one of pure shear, while for material $M 2$ the smallest localization length appears to be near values of $\phi=0.431$. We also observe that in both cases the 
localized zone length $L_{c}$ varies very little with the strain concentration parameter for values of $\phi$ corresponding to the smallest possible $L_{c}$. Finally, we would like to point out the influence of the type of gradient term added to the initial strain energy density (here $w_{\mathrm{BK}}$ ) on $L_{c}$ by noting the order of magnitude difference between the $M 1$ and M2 models.

\section{Acknowledgements}

The support of the Solid Mechanics Program of the National Science Foundation and the $M M$ program of MTU is gratefully acknowledged. The results reported herein were essentially obtained in July 1984 when NT was visiting ECA at Michigan Tech. and initially printed as an MM Report No. 6 in August 1984.

\section{References}

[1] E.C. Aifantis and J.B. Serrin, The Mechanical Theory of Fluid Interfaces and Maxwell's Rule. J. Coll. and Interface Science 96 (1983) 517-529.

[2] E.C. Aifantis and J.B. Serrin, Equilibrium Solutions in the Mechanical Theory of Fluid Microstructures. J. Coll. and Interf. Sci. 96 (1983) 530-547.

[3] E.C. Aifantis, Some Thoughts on Degrading Materials, in: NSF Workshop on Mechanics of Damage and Fracture, Eds. S.N. Atluri and J.E. Fitzgerald. pp. 1-12, Atlanta (1982).

[4] a. E.C. Aifantis, Dislocation Kinetics and the Formation of Deformation Bands, in: Defects Fracture and Fatigue, Eds. G.C. Sih and J.W. Provan. pp. 75-84, Martinus-Nijhoff (1983).

b. E.C. Aifantis, Towards a Continuum Approach to Dislocation Patterning, in: Dislocations in Solids-Recent Advances, AMD-63, Ed. X. Markenscoff. pp. 23-33, ASME (1984).

c. E.C. Aifantis, "On Dislocation Patterning", in: Dislocations in Solids, Eds. H. Suzuki, T. Ninomiya, K. Sumino, and S. Takeuchi. pp. 41-47, Univ. of Tokyo Press (1985).

[5] E.C. Aifantis, Microscopic Processes and Macroscopic Response, in: Mechanics of Engineering Materials, Eds. C.S. Desai and R.H. Gallagher. pp. 1-22, John Wiley (1984).

[6] a. E.C. Aifantis, On the Mechanics of Modulated Structures, in: Modulated Structure Materials, NATO ASI Series 83, Ed. T. Tsakalakos. pp. 357-385, Martinus-Nijhoff (1984).

b. E.C. Aifantis, Continuum Models for Dislocated States and Media with Microstructures, in: The Mechanics of Dislocations, Eds. E.C. Aifantis and J.P. Hirth. pp. 127-146, ASM, Metals Park (1985).

[7] E.C. Aifantis, On the Microstructural Origin of Certain Inelastic Models. Transactions of ASME, $J$. Engng. Mat. Tech. 106 (1984) 326-330.

[8] J. Hadamard, Lecons sur la Propagation des Ondes et les Equations de l'Hydrodynamique. Chap. 6, Paris (1903).

[9] T.Y. Thomas, Plastic Flow and Fracture in Solids. Academic Press (1961).

[10] R. Hill, Acceleration Waves in Solids. J. Mech. Phys. Solids 10 (1962) 1-16.

[11] J. Mandel, Conditions de Stabilité et Postulat de Drucker, in: Rheology and Soil Mechanics, Eds. J. Kravtchenko and P.M. Sirieys. pp. 58-68, Springer (1966).

[12] J.K. Knowles and E. Sternberg, On the Failure of Ellipticity of the Equations for Finite Elastostatics Plane Strain. Arch. Rat. Mech. Analysis 63 (1977) 321-336.

[13] J.R. Rice, The Localization of Plastic Deformation, in: Theoretical and Applied Mechanics (Proceedings of the 14th I.U.T.A.M. Conference, Delft, August 30-Sept. 4 (1976), Ed. W.T. Koiter, (1976) 207-220, North-Holland.

[14] Marciniak, Z. and K. Kuczynski, Limit Strains in the Process of Stretch Forming Sheet Metal. Int. $J$. Mech. Sciences 9 (1967) 609-625.

[15] V. Tvergaard, A. Needleman and K.K. Lo, Flow Localization in the Plane Strain Tensile Test. J. Mech. Phys. Solids 29 (1981) 115-142.

[16] E.C. Aifantis, Maxwell and van der Waals Revisited, in: Phase Transformations in Solids, Ed. T. Tsakalakos. MRS Vol. 21 (1984) 37-49, North-Holland. 
[17] V. Alexiades and E.C. Aifantis, On the Thermodynamic Theory of Fluid Interfaces: Infinite Intervals, Equilibrium Solutions, and Minimizers. J. Coll. Interf. Sci. 111 (1986) 119-132.

[18] R.A. Toupin, Elastic Materials with Couple-stresses. Arch. Rat. Mech. Anal. 11 (1962) 385-414.

[19] R.A. Toupin, Theories of Elasticity with Couple-stress. Arch. Rat. Mech. Anal. 17 (1964) 85-112.

[20] R.D. Mindlin, Micro-structure in Linear Elasticity. Arch. Rat. Mech. Anal. 16 (1964) 51-78.

[21] R.D. Mindlin, Second Gradient of Strain and Surface Tension in Linear Elasticity. Int. J. Solids Struct. 1 (1965) 417-438.

[22] A.E. Green and R.S. Rivlin, Multipolar Continuum Mechanics. Arch. Rat. Mech. Anal. 17 (1964) 113-147.

[23] J.K. Knowles and E. Sternberg, On the Failure of Ellipticity and the Emergence of Discontinuous Deformation Gradients in Plane Finite Elastostatics. J. Elasticity 8 (1978) 329-379.

[24] R. Hill and J.W. Hutchinson, Bifurcation Phenomena in the Plane Tension Test. J. Mech. Phys. Solids. 23 (1975) 239-264.

[25] J.E. Dunn and J. Serrin, On the Thermodynamics of Interstitial Working, Arch. Rat. Mech. Anal. 88 (1985) 95-133.

[26] M. Silhavy and E.C. Aifantis, Generalized Thermomechanics of Continua-I, preprint.

[27] C. Truesdell and R.A. Toupin, The Classical Field Theories, in Encyclopedia of Physics Vol. 3/1, Springer (1960).

[28] J.J. Cross, Mixtures of Fluids and Isotropic Solids. Arch. Mech. 25 (1973) 1024-1039.

[29] R.M. Bowen, Theory of Mixtures, in Continuum Physics, Vol. III, Ed. A.C. Eringen, pp. 1-127, Academic Press (1976).

[30] G. Ficherra, Existence Theorems in Elasticity, in Encyclopedia of Physics Vol. VIa/2, Springer (1970).

[31] P.J. Blatz and W.L. Ko, Application of Finite Elastic Theory to the Deformation of Rubbery Materials Trans. Soc. Rheology Vol. 6, (1962) 223-251.

[32] J.K. Knowles and E. Sternberg, On the Ellipticity of the Equations of Nonlinear Elastostatics for a Special Material. J. Elasticity 5 (1975) 334-341. 